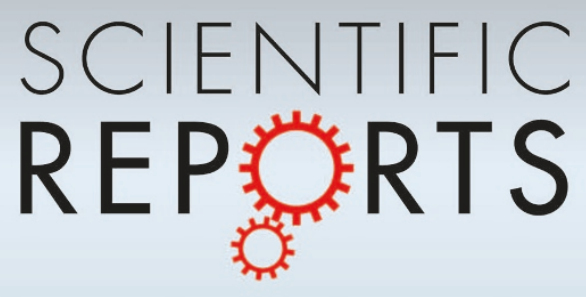

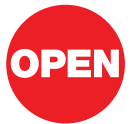

SUBJECT AREAS:

MAGNETIC MATERIALS

AND DEVICES

APPLIED PHYSICS

MATERIALS PHYSICS

ELECTRONIC MATERIALS AND

DEVICES

Received

30 May 2012

Accepted

22 August 2012

Published

18 September 2012

Correspondence and requests for materials should be addressed to

F.X. (Faxian@iastate. edu) or K.L.W. (wang@ ee.ucla.edu)

\title{
Quantum Capacitance in Topological Insulators
}

\author{
Faxian Xiu', Nicholas Meyer' ${ }^{1}$, Xufeng Kou ${ }^{2}$, Liang He${ }^{2}$, Murong Lang ${ }^{2}$, Yong Wang ${ }^{3}$, Xinxin $\mathrm{Yu}^{2}$, \\ Alexei V. Fedorov ${ }^{4}$, Jin Zou ${ }^{5} \&$ Kang L. Wang ${ }^{2}$
}

\begin{abstract}
'Department of Electrical and Computer Engineering, lowa State University, Ames, USA, ${ }^{2}$ Department of Electrical Engineering, University of California, Los Angeles, California 90095, USA, ${ }^{3}$ State Key Laboratory of Silicon Materials and Center for Electron Microscopy, Department of Materials Science and Engineering, Zhejiang University, Hangzhou, 310027, China, ${ }^{4}$ Advanced Light Source Division, Lawrence Berkeley National Laboratory, 1 Cyclotron Road, Berkeley, California, 94720, USA, ${ }^{5}$ Materials Engineering and Centre for Microcopy and Microanalysis, The University of Queensland, Brisbane QLD 4072, Australia.
\end{abstract}

Topological insulators show unique properties resulting from massless, Dirac-like surface states that are protected by time-reversal symmetry. Theory predicts that the surface states exhibit a quantum spin Hall effect with counter-propagating electrons carrying opposite spins in the absence of an external magnetic field. However, to date, the revelation of these states through conventional transport measurements remains a significant challenge owing to the predominance of bulk carriers. Here, we report on an experimental observation of Shubnikov-de Haas oscillations in quantum capacitance measurements, which originate from topological helical states. Unlike the traditional transport approach, the quantum capacitance measurements are remarkably alleviated from bulk interference at high excitation frequencies, thus enabling a distinction between the surface and bulk. We also demonstrate easy access to the surface states at relatively high temperatures up to $60 \mathrm{~K}$. Our approach may eventually facilitate an exciting exploration of exotic topological properties at room temperature.

$\mathrm{n}$ recent years, the discovery of a new class of topological states of matter, known as time-reversal invariant $\mathrm{Z}_{2}$ topological insulators (TIs), has generated considerable excitement in condensed matter physics. These materials are characterized by a bulk insulating energy gap and gapless edges or surface states which are topologically protected because an electron's spin is locked to its momentum due to a strong spin-orbit interaction $^{1-14}$. Theory predicts that the unique surface states hosting Dirac fermions are robust and immune to defects, non-magnetic impurities and other perturbing influences in their environment, leading to potential non-dissipative applications $s^{3,14-16}$. To experimentally determine the macroscopic properties of the Dirac fermions and intentionally gain a control of the helical states, substantial efforts have been made in improving material quality using topological thin films ${ }^{17-22}$, bulk crystals $\mathrm{s}^{5,13,23}$ and nanostructures ${ }^{24-26}$ and in developing sensitive approaches for revealing surface transport and spin textures using angle-resolved photoemission (ARPES $)^{6,13,17,21,27,28}$, scanning tunneling microscopy $(\mathrm{STM})^{8,29}$, low-temperature transport ${ }^{5,23,30-33}$, and optical polarizations ${ }^{10}$. While significant progress has been achieved in identifying Dirac fermions via Shubnikov-de Haas $(\mathrm{SdH})$ oscillations ${ }^{5,31}$ and ambipolar field effects ${ }^{30}$ at low temperatures, experimental realization of exotic new physical phenomena arising from the surface states has been hindered by parallel bulk conductions due to naturally occurring defects and residual carrier doping ${ }^{13,21,28}$. Thus, developing new methods of separating the contributions from the bulk and surface states becomes one of the most important tasks in the field, particularly in the high temperature regime where bulk carriers are activated ${ }^{25}$.

Quantum capacitance has advantages over traditional transport measurements because it allows us to directly probe the surface density of states (DOS), whereas the latter are more complicated and sensitive to scattering details ${ }^{34}$. Quantum capacitance is described by the equation $C_{Q}=e^{2} D(E)$, where $D(E)$ represents the DOS ${ }^{34-36}$. Thus, a simple measurement of quantum capacitance provides a quantitative description of the DOS at the Fermi energy $^{34,36-43}$. Despite its capability of directly probing electronic compressibility at high temperatures ${ }^{34}$, this concept has never been applied to TIs, which is in part attributed to the lack of high-quality crystals, on which a large atomically flat surface is required for detectable quantum capacitance. As the material quality has been progressively improved recently ${ }^{5,17,18,21-24,30-32,44}$, the quantum capacitance measurements become especially important as they can potentially solve today's intriguing challenge of high-temperature surface-state detection and may subsequently lead to the exploration of quantized anomalous and quantum spin Hall effects ${ }^{1,14-16,45}$. 
Here, we report on an observation of quantum capacitance using a topological capacitor device fabricated on $\mathrm{Bi}_{2} \mathrm{Se}_{3}$ thin films. Under external magnetic fields, by varying gate voltages, we observed pronounced capacitance oscillations associated with the creation of quantized Landau levels. Remarkably, by changing the $a c$ frequency during the measurements, the amplitudes of the oscillations can be systematically tuned. As a result, high-frequency excitations are observed to sufficiently resolve the surface states up to $60 \mathrm{~K}$. Our theoretical simulations further suggest that the different frequency responses of carriers from the surface and bulk states may account for the distinction of the surface states at high frequencies.

\section{Results}

Growth of $\mathrm{Bi}_{2} \mathrm{Se}_{3}$ thin films. $\mathrm{Bi}_{2} \mathrm{Se}_{3}$ thin films were grown on $\mathrm{Si}$ (111) substrates using an ultra-high vacuum molecular-beam epitaxy (MBE) system. Figure la presents a large-scale atomic force microscopy (AFM) image of an as-grown $\mathrm{Bi}_{2} \mathrm{Se}_{3}$ film with a thickness of $10 \mathrm{~nm}$, exhibiting terraces over $500 \mathrm{~nm}$ in size. The surface consists of triangle-shaped terraces and steps $(\sim 1 \mathrm{~nm}$ in height, Supplementary Fig. S1), indicative of a hexagonal crystal structure along the (111) direction ${ }^{18}$. Real-time reflection high-energy electron diffraction (RHEED) was used to monitor the in-situ growth dynamics with the electron beam incident to the $[11 \overline{2} 0]$ direction. The sharp streaky lines indicate a $2 \mathrm{D}$ growth mode and a flat surface morphology (Fig. 1b). To verify the existence of the surface states, high-resolution ARPES experiments along the $\vec{\Gamma}-\overrightarrow{\mathrm{K}}$ direction were performed at $64 \mathrm{~K}$ (Fig. 1c). A nearly linear $E$ - $k$ dispersion is observed near the Dirac point. The inset presents a curvature analysis of the raw data for a clear visualization of the band dispersion. The Fermi level is located $\sim 0.4 \mathrm{eV}$ above the Dirac cone, suggesting a very high bulk concentration. Such a large binding energy of the Dirac point in the $\mathrm{Bi}_{2} \mathrm{Se}_{3}$ thin film is believed to originate from the creation of Se vacancies by Se out-diffusion ${ }^{22,31,46}$ during the preannealing process, as the film was annealed at $200^{\circ} \mathrm{C}$ for 2 hours before the ARPES experiments. Other widely observed effects such as band bending ${ }^{22,31,47}$ and photoemission-induced gating ${ }^{48}$ may not play significant roles in our film because of the large bulk density after annealing. The surface states must also be examined via other techniques such as low-temperature transport and STM, where a heating process and period can be minimized during sample preparation. As anticipated, the transport experiments exhibit clear SdH oscillations (Supplementary Fig. S2). Unlike the ARPES results, the Fermi level was estimated to be $53 \pm 5 \mathrm{meV}$ above the Dirac point, consistent with the value of $70 \pm 20 \mathrm{meV}$ obtained from the STM experiments (Supplementary Fig. S3). The location of the Fermi level relative to the Dirac point can be further verified using the quantum capacitance measurements, as discussed later.

Capacitor device structure. To perform accurate capacitance measurements, an appropriate design of the device structure is essential. Capacitance measurements are extremely intricate because they involve multiple components that respond to different frequencies. To minimize the undesired components, the structure of the devices must be as simple as possible. Figure $1 \mathbf{d}$ presents a schematic drawing of a capacitor using a $10 \mathrm{~nm}$-thick $\mathrm{Bi}_{2} \mathrm{Se}_{3}$ thin film as the channel layer. A metal stack of $\mathrm{Fe} / \mathrm{Al}(20 / 100 \mathrm{~nm})$ is deposited on the top surface of the $\mathrm{Bi}_{2} \mathrm{Se}_{3}$ thin film to act as the source and drain contacts. A thin layer of $\mathrm{Al}_{2} \mathrm{O}_{3}(15 \mathrm{~nm})$ is used as the gate oxide.

The total gate capacitance $\left(C_{T}\right)$ can be modeled as a series of oxide capacitance $\left(C_{O X}\right)$, quantum capacitance $\left(C_{Q}\right)$, depletion/accumulation capacitances $\left(C_{D / A}\right)$, and bulk capacitance $\left(C_{B}\right)$, that is, $1 / C_{T}=1 /$ $C_{O X}+1 / C_{Q}+1 / C_{D / A}+1 / C_{B}$. Note that there are other parasitic capacitances associated with the source and drain; however, by performing a measurement on a device with identical geometry but without the topological insulator channel, these parasitic capacitances are negligible compared with the top-gate capacitance. To a first approximation, we may thus neglect the effect due to the parasitic capacitances. The total gate capacitance is primarily dominated by the smallest capacitance component in the system, i.e., the smallest magnitude among $C_{O X}, C_{Q}, C_{D / A}$ and $C_{B}$. To gain further insight into the underlying physics, the concepts of $C_{Q}$ and $C_{D / A}$ must be clarified. $C_{Q}$ represents the quantum capacitance associated with the topological surface DOS, while $C_{D / A}$ describes a depletion $\left(C_{D}\right)$ or an a

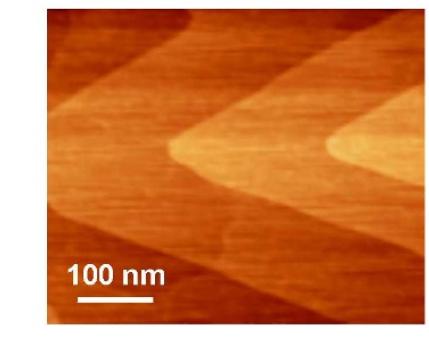

b

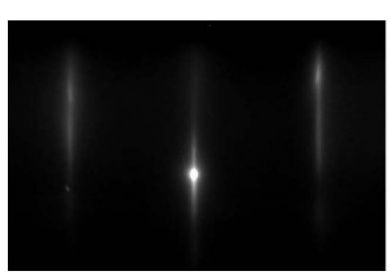

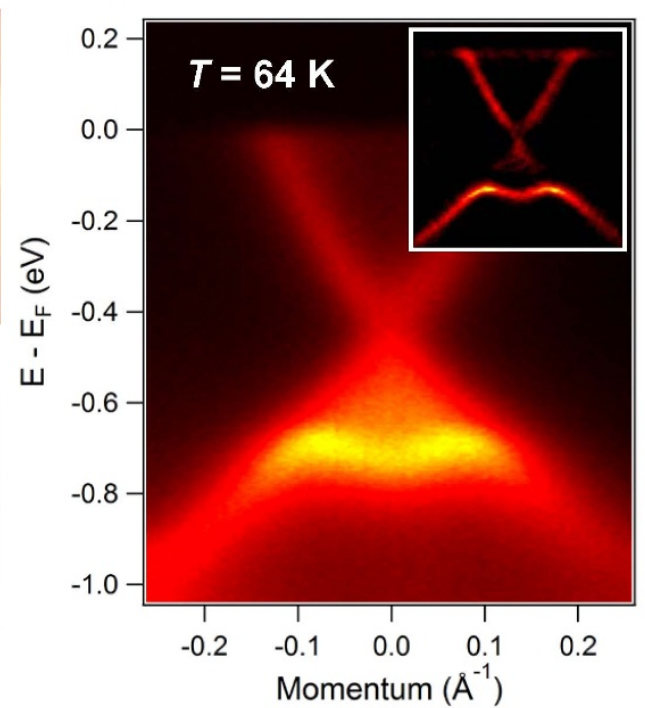

d

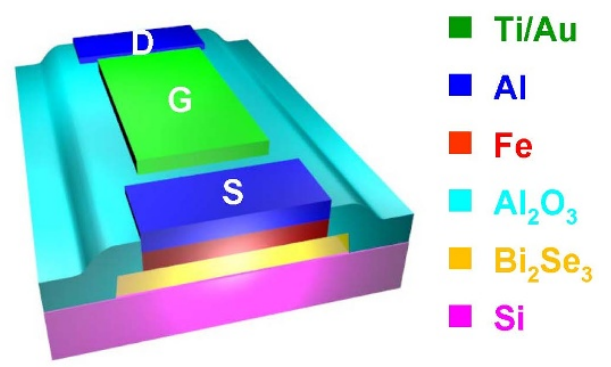

e

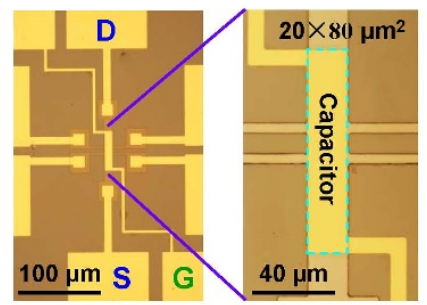

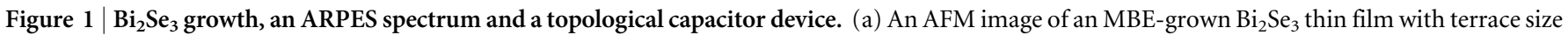
exceeding $500 \mathrm{~nm}$. (b) A streaky RHEED pattern along the [11 $\overline{2} 0$ ] direction of the as-grown surface of $\mathrm{Bi}_{2} \mathrm{Se}_{3}$, indicating a 2-D growth mode. (c) An ARPES spectrum of the film along the $\vec{\Gamma}-\overrightarrow{\mathrm{K}}$ direction at $T=64 \mathrm{~K}$. The inset shows the curvature of the raw data for better visualization of the band

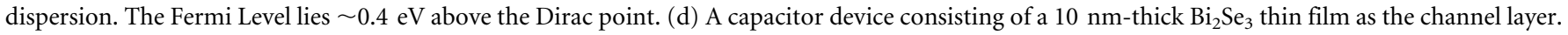
The source-drain contacts were composed of Fe/Al $(20 \mathrm{~nm} / 100 \mathrm{~nm}$ ), while the gate electrode consists of Ti/Au, $10 \mathrm{~nm} / 100 \mathrm{~nm}$. (e) An optical

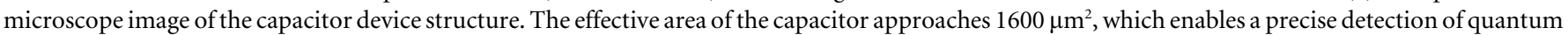
capacitance from the $\mathrm{Bi}_{2} \mathrm{Se}_{3}$ top surface. 
a

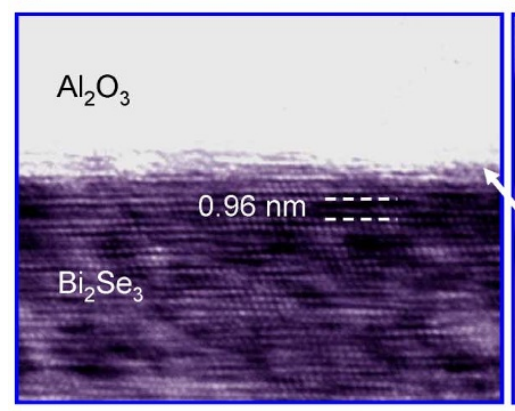

b

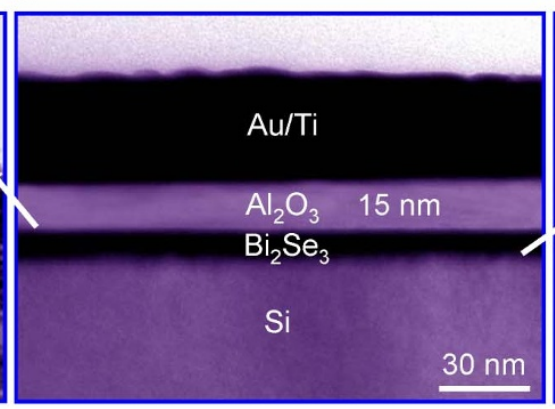

C

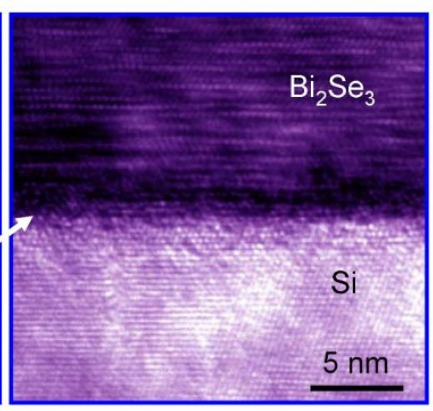

Figure $2 \mid$ Cross-section TEM images of $\mathrm{B}_{2} \mathrm{Bi}_{3}$ capacitor device. (a) A high-resolution TEM picture of the top surface of $\mathrm{Bi}_{2} \mathrm{Se}_{3}$ with a thickness of approximately10 nm. $\mathrm{Al}_{2} \mathrm{O}_{3}-\mathrm{Bi}_{2} \mathrm{Se}_{3}$ has an atomically sharp interface, which helps to preserve the topological states. The lattice spacing between the (0003) planes is measured to be $0.96 \mathrm{~nm}$. (b) A low-magnification TEM image of the entire cross-section structure of the $\mathrm{Bi}_{2} \mathrm{Se}_{3}$ capacitor device. A thin and high-quality $\mathrm{Al}_{2} \mathrm{O}_{3}$ film of $15 \mathrm{~nm}$ is used to increase the oxide capacitance, making it possible to observe quantum capacitance from the $\mathrm{Bi}_{2} \mathrm{Se}_{3}$ top surface. (c) A high-resolution TEM for the bottom interface between $\mathrm{Bi}_{2} \mathrm{Se}_{3}$ and $\mathrm{Si}$. An atomically sharp interface is also observed.

accumulation capacitance $\left(C_{A}\right)$ during device operation. Under a depletion mode, electrons are depleted within a depth of $w$. Depending on the applied voltage, the depletion capacitance $C_{D}$ changes its magnitude owing to the dynamic variation of $w$ (ref. 49). Thus, in this scenario, a direct comparison of the magnitude of the different capacitive components is challenging without constructing numerical simulation models. However, in contrast, under an accumulation mode, the capacitance $C_{A}$ becomes much larger than the other components. In particular, as the gate bias increases, a steady rise of the carrier density produces a large $C_{A}$ according to the equation $C_{A}=\left(\varepsilon_{T I} / \sqrt{2} \lambda_{n}\right)\left(N_{S} / N_{D}\right)^{1 / 2}$ (refs. 37,49,50), where $\varepsilon_{\mathrm{TI}}$ is the dielectric constant of $\mathrm{Bi}_{2} \mathrm{Se}_{3}, \lambda_{n}$ is the extrinsic Debye length, $N_{s}$ is the carrier density in the inversion layer and $N_{D}$ is the density of ionized donors. Consequently, as the device enters a saturation regime, the total capacitance can be simplified as $1 / C_{T}=1 / C_{O X}+1 /$ $C_{\mathrm{Q}}+1 / C_{B}$ with the bulk capacitance $C_{B}$ being an unknown parameter. By systematically increasing the measurement frequency, the $C_{B}$ component can be easily eliminated based upon different frequency response of the surface and bulk carriers ${ }^{49,51-54}$, as elaborated in the Simulation and Discussion section. Therefore, the extraction of $C_{Q}$ becomes immediately feasible under the accumulation mode, where the oxide capacitance can be calculated by $C_{O X}=\varepsilon_{r} \varepsilon_{0} / d\left(d\right.$ is the thickness of $\left.\mathrm{Al}_{2} \mathrm{O}_{3}\right)$.

Figure 1e shows an optical microscope image of the capacitor device structure. We performed the Hall and capacitance measurements with a Hall bar geometry. The effective area of the capacitor is measured to be approximately $1600 \mu \mathrm{m}^{2}$. The large area ensures a high signal-to-noise ratio and a good estimation of the quantum capacitance from the $\mathrm{Bi}_{2} \mathrm{Se}_{3}$ top surface. The cross-section of the practical Hall bar devices was examined using a high-resolution transmission electron microscopy (HRTEM). As illustrated in Fig. $2 \mathbf{b}$, the device consists of $15 \mathrm{~nm}$ of $\mathrm{Al}_{2} \mathrm{O}_{3}$ and $10 \mathrm{~nm}$ of $\mathrm{Bi}_{2} \mathrm{Se}_{3}$. The interfaces at the top and bottom of the $\mathrm{Bi}_{2} \mathrm{Se}_{3}$ thin film both have atomically sharp heterojunctions (Fig. 2a and 2c). The lattice spacing is observed to be $0.96 \mathrm{~nm}$, consistent with other reports ${ }^{18,24}$.

Quantum capacitance in $\mathbf{B i}_{2} \mathrm{Se}_{3}$ thin films. Figure 3 plots the total capacitance as a function of gate voltage, magnetic field and a

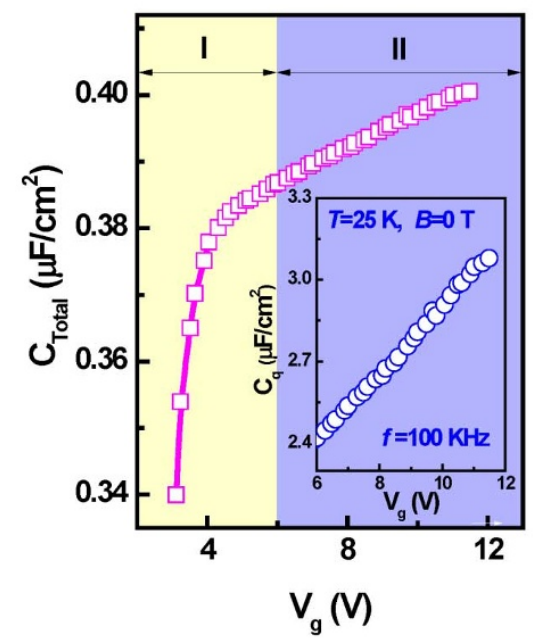

b

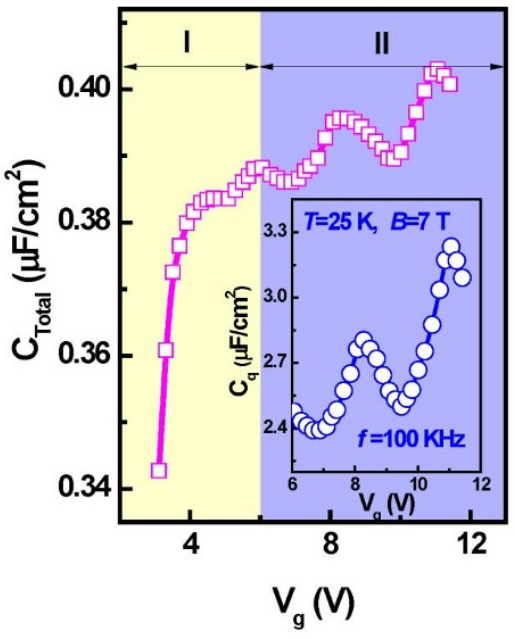

c

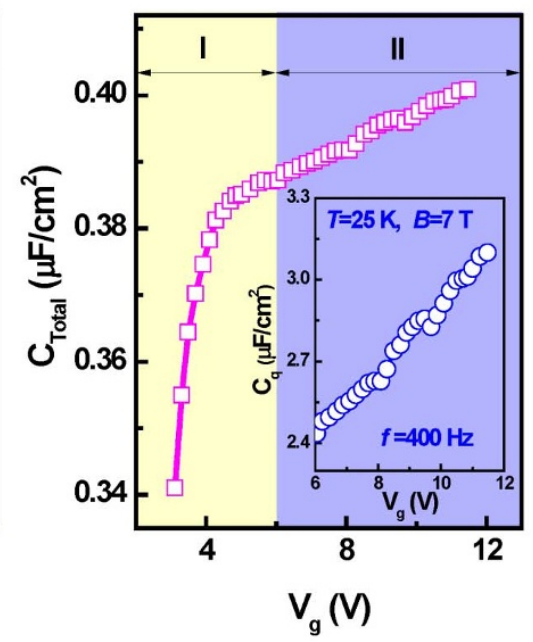

Figure 3 Extraction of quantum capacitance in $\mathrm{Bi}_{2} \mathrm{Se}_{3}$ capacitors. (a) The total capacitance as a function of gate voltage at $T=25 \mathrm{~K}, B=0 \mathrm{~T}$ and $f=100 \mathrm{KHz}$. The capacitance values in regimes I (yellow) and II (light purple) are dominated by the bulk and the TI top surface, respectively. The continuing increase in the total capacitance in regime II suggests the presence of quantum capacitance $\left(C_{Q}\right)$, which can be directly extracted based on a series capacitor model where $1 / C_{T}=1 / C_{O X}+1 / C_{Q}$. The resultant $C_{Q}$ is shown in the inset. (b) The total capacitance as a function of gate voltage at $T=25 \mathrm{~K}, B=7 \mathrm{~T}$ and $f=100 \mathrm{KHz}$. Capacitance oscillations were observed under an external magnetic field of $7 \mathrm{~T}$, suggesting the creation of Landau levels from the surface states. The inset presents the extracted oscillatory $C_{Q}$. (c) The total capacitance as a function of gate voltage at $T=25 \mathrm{~K}, B=7 \mathrm{~T}$ and $f=400 \mathrm{~Hz}$. As compared with (b) under low-frequency excitation, the quantum oscillations tend to diminish. These results indicate that detection of the surface states can be achieved by applying high frequencies during the $C$ - $V$ measurements. 
measurement frequency in a $\mathrm{Bi}_{2} \mathrm{Se}_{3}$ capacitor at $25 \mathrm{~K}$. In general, two regimes are observed for the gate voltage ranging from 0 to $12 \mathrm{~V}$. In regime I, the total capacitance is dominated by the depletion capacitance; however, the quantum capacitance cannot be extracted because of the uncertainty of the depletion component ${ }^{49}$. When the gate voltage further increases, the total capacitance tends to saturate (Fig. 3a and 3c), the quantum capacitance not only contributes a small correction to the total gate capacitance but also begins to dominate over the other components in this regime. The system can be then simplified as $1 / C_{T}=1 / C_{O X}+1 / C_{Q}$ (the term $C_{B}$ is eliminated at high frequencies as shown in Fig. 4); here, the oxide capacitance is calculated to be $0.46 \mu \mathrm{F} / \mathrm{cm}^{2}$ by taking $d=15 \mathrm{~nm}$ and $\varepsilon_{r}=7.8$ (extracted from the control sample, Supplementary Fig. S4).

For a traditional MOS capacitor, under the accumulation mode, the capacitance should saturate at a constant value corresponding to the oxide capacitance (Supplementary Fig. S4, a regular MOS capacitor without $\mathrm{Bi}_{2} \mathrm{Se}_{3}$ ). For a $\mathrm{Bi}_{2} \mathrm{Se}_{3}$ capacitor, however, the total capacitance increases continuously as the gate scans from 6 to $12 \mathrm{~V}$ (Fig. 3a). The gradual increase of the total capacitance must originate from an additional capacitive component (Supplementary Fig. S5), which could be attributed to the quantum capacitance of the surface states. To verify this presumption, we applied a magnetic field of $7 \mathrm{~T}$ perpendicular to the sample surface to observe the possible quantization of Landau levels. Indeed, significant capacitance oscillations were resolved (Fig. 3b), suggesting that the quantum capacitance becomes dominant and changes significantly with varying gate voltage and magnetic field ${ }^{34}$. It is speculated that during the capacitancevoltage $(C-V)$ measurements, the Fermi level is shifted because of the gating effect ${ }^{33}$ : as it moves across each discrete Landau level, the quantum capacitance becomes oscillatory (Fig. $3 \mathbf{b}$ inset, $C_{Q}$ vs. $V_{g}$ ). This analysis is essentially the same as for the case of SdH oscillations ( $R_{x x}$ vs. $B$, where $R_{x x}$ represents longitudinal resistance) ${ }^{42,55}$, and the capacitance results are nearly identical to the transport measurements but with a much higher temperature. By subtracting the oxide capacitance, $C_{Q}$ from the topological surface is retrieved, as depicted in all three insets of Fig. 3. The obtained $C_{Q}$ is on the order of $2 \mu \mathrm{F} /$ $\mathrm{cm}^{2}$, reasonably near the theoretical calculation (Supplementary Fig. S5). To explore the effect of frequency on the surface states, the excitation frequency was reduced from $100 \mathrm{KHz}$ to $400 \mathrm{~Hz}$. The quantum oscillations, however, diminished rapidly, exhibiting a noisy spectrum without evident traces of oscillations (Fig. $3 \mathrm{c}$ inset). These observations imply that the detection of the surface states can be achieved by applying high frequency excitations during the $C-V$ measurements.

Figure 4 shows a comprehensive analysis of the quantum capacitance in $\mathrm{Bi}_{2} \mathrm{Se}_{3}$ for different experimental settings. Figure $4 \mathrm{a}$ demonstrates the magnetic field dependence of the total capacitance at $100 \mathrm{KHz}$ and $25 \mathrm{~K}$. When the magnetic field increases from 3 to 9 $\mathrm{T}$, the low-index Landau levels with large interleave spacing progressively move across the Fermi level, reminiscent of typical SdH oscillations from transport measurements ${ }^{42,55}$ (see also Supplementary Fig. S2). Figure $4 \mathrm{~b}$ demonstrates a clear trend of frequency-dependent quantum oscillations, where high frequency excitations resolve the surface oscillations quite well. Figures $4(\mathbf{e}, \mathbf{f})$ and $4(\mathbf{i}, \mathbf{j})$ present similar results but at higher temperatures of 35 and $50 \mathrm{~K}$, respectively. Note that the frequency can have a significant effect on the distinction of the surface from the bulk ${ }^{49,51-54}$, presumably because of the dramatic difference in carrier mobility: in the bulk, the electron mobility is approximately $380 \mathrm{~cm}^{2} / \mathrm{V} \mathrm{s}$, while on the surface the electron mobility reaches over $10,000 \mathrm{~cm}^{2} / \mathrm{V}$ s (Supplementary Figs. S2 and S6). Such a large difference enables a fast response from the surface states, analogous to the fast response of high-mobility electrons in graphene (in a gigahertz range) ${ }^{56}$; as a comparison, the bulk carriers cannot follow the high frequency excitations, resulting in a "filtering" effect ${ }^{49,51}$. More importantly, the surface states can be clearly distinguished at approximately $60 \mathrm{~K}$ (Fig. 4c and
Supplementary Fig. S7), much beyond the capability of the low-temperature transport, where movable carriers travel along horizontal channels and are extremely sensitive to bulk properties (scattering details $)^{34}$. In addition, quantum capacitance probes both the extended and localized states, whereas electron transport can only investigate the former one ${ }^{34}$.

To better understand the physics behind this phenomenon, we performed a quantitative analysis of the $\mathrm{SdH}$ oscillations. Figure $4 \mathrm{c}$ demonstrates the temperature dependence of the total capacitance when the magnetic field and frequency are set to $7 \mathrm{~T}$ and $100 \mathrm{KHz}$, respectively. The surface states are persistent up to $60 \mathrm{~K}$. Three peaks are identified, corresponding to the generation of three Landau levels in the system. The first derivative of the total capacitance was also taken to remove the background parasitic capacitances (Fig. 4d). The three peaks from $\mathrm{P} 1$ to $\mathrm{P} 3$ have a common $90^{\circ}$ phase shift compared with those in Fig. 4c. Now, we can take the amplitude of the capacitance oscillations - note that the $\mathrm{Al}_{2} \mathrm{O}_{3}$ capacitance $C_{O X}$ is removed - and apply $\Delta C(T) / \Delta C(0)=\lambda(T) / \sinh (\lambda(T))$, where the thermal factor is given by $\lambda(T)=2 \pi^{2} k_{B} T m_{c y c l} /(\hbar e B)($ refs. 5,23). Here, $m_{\text {cycl }}$ denotes the cyclotron mass, $k_{B}$ is Boltzmann's constant, and $\hbar$ is the reduced Plank's constant. By taking the conductivity oscillation amplitude and performing the best fit to the equation of $\Delta C(T)$ / $\Delta C(0)$ (Fig. $4 \mathrm{~g}$ ), $m_{\text {cycl }}$ is calculated to be $0.079 \mathrm{~m}_{0}, 0.091 \mathrm{~m}_{0}$, and $0.102 \mathrm{~m}_{0}$ at gate voltages of $5.9 \mathrm{~V}(\mathrm{P} 1), 8 \mathrm{~V}(\mathrm{P} 2)$ and $10.9 \mathrm{~V}(\mathrm{P} 3)$, respectively (Fig. $4 \mathrm{~g}$ (inset)). These values are reasonably close to those extracted from the transport measurements $\left(0.07 \mathrm{~m}_{0}\right.$, Supplementary Fig. S2). Here, $\mathrm{m}_{0}$ is the electron rest mass. Because the cyclotron mass is given by $m_{c y c l}=E_{F} / V_{F}^{2}$ (ref. 5), where $E_{F}$ and $V_{F}$ are the Fermi level and the Fermi velocity, both $E_{F}$ and $V_{F}$ can be precisely obtained given $m_{c y c l} V_{F}=\hbar k_{F}$ (ref. 23). It is noted that the Fermi level increases from 61.5 to $79.4 \mathrm{meV}$ when the gate voltage is varied from +5.9 to $+10.9 \mathrm{~V}$ (Fig. $4 \mathrm{~h}$ ), exhibiting the clear gate modulation behavior of the surface states. The $k_{F}$ value can also be obtained in the range of $0.025-0.033 \AA^{-1}$, consistent with the transport results (Supplementary Fig. S2).

We performed a series of sample rotations under a constant magnetic field of $7 \mathrm{~T}$ to identify the nature of the quantum oscillations (Fig. $4 \mathbf{k}$ and $4 \mathbf{i})^{5,23}$. As the system is rotated from 0 to $50^{\circ}$, the amplitude of the oscillations dampens and the oscillation frequency (peak interval) changes, depending on the magnitude of $B_{\perp}$ (Fig. $4 \mathbf{k}$ inset), which signifies the typical 2-D characteristics of the topological surface states ${ }^{5}$. The relationship between the carrier density $n$ and the Fermi vector $k_{F}$ is the same for both quadratic (2-DEG) and linear (TI surface state) $E-k$ dispersion relations and is given by $n \propto k_{F}^{2} / \pi$ (refs. 39,57). Accordingly, the period of SdH oscillations for both 2 -DEG and TI surface states also exhibit similar behavior given the Onsager relation of $f=1 / \Delta(1 / B)=2 \hbar \pi n / q($ ref. 5$)$. Therefore, it is difficult to distinguish the surface states from 2-DEG through the conventional magneto-transport measurements, which can only probe the carriers that transport through the channel. However, unlike the transport approach, the quantum capacitance method can circumvent this challenge by providing a direct extraction of an important quantity, $d n / d V_{a}$ (ref. 39, $V_{a}$ - the voltage dropped on the TI surface), which explicitly describes the surface states. By carefully examining their $E-k$ relations and $\operatorname{DOS}^{39,57}$, i.e., $E(k)=$ $\hbar v_{F}|k| \& \mathrm{~g}_{T I}(E)=g|E| / 2 \pi\left(\hbar v_{F}\right)^{2}$ for the surface states, $E(k)=\hbar^{2} k^{2}$ $/ 2 m \& \mathrm{~g}_{2 D E G}(E)=m N(E) / \pi \hbar^{2}$ for 2-DEG, one can theoretically derive and simulate their capacitances, as demonstrated in Supplementary Figs. S5 and S8. Here, $g=g_{s} g_{v}$ is the degeneracy factor, considering both the spin and energy band degeneracies for the TI surface states. The quantum capacitance associated with the surface states strongly depends on the density of states, similar to that of graphene ${ }^{34,36,37}$. By contrast, for 2-DEG the capacitance is saturated at a constant value irrespective of the change in $V_{a}$ (Supplementary Fig. S8). Therefore, the observed quantum oscillations in Figs. 3 and 4 are attributed to the surface states instead of 2-DEG, which is also 
a

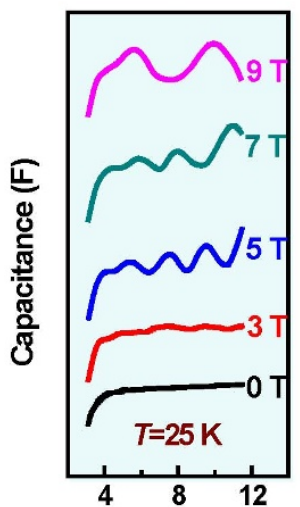

e

Gate Voltage (V)

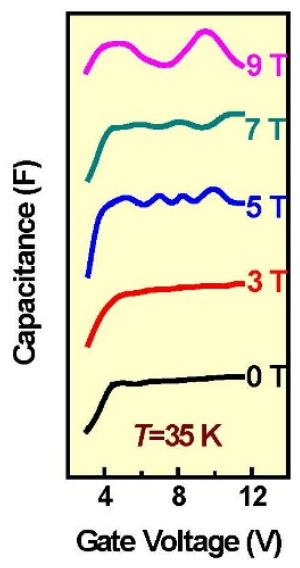

i

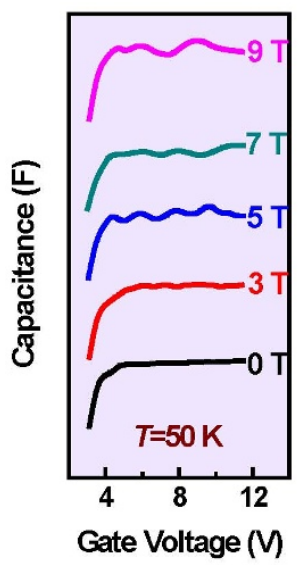

b
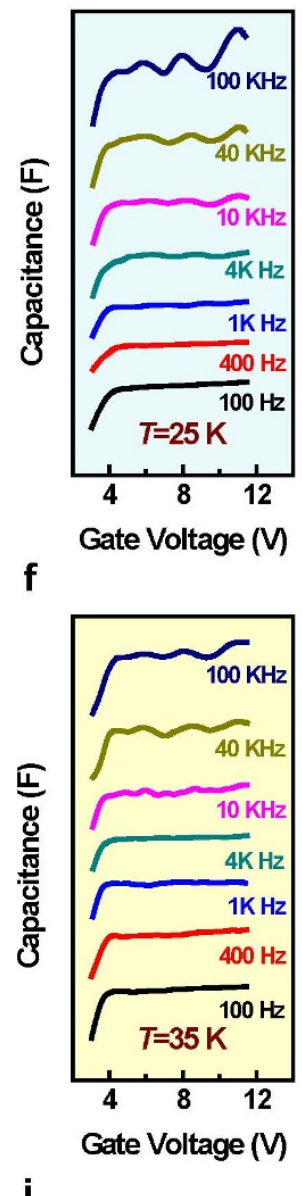

j

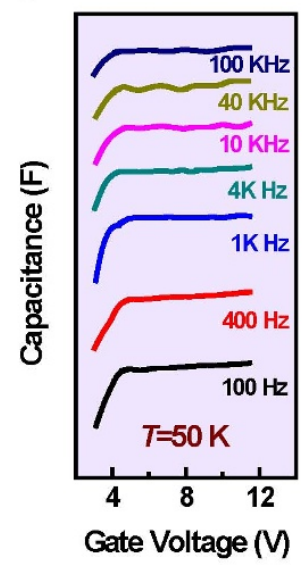

c

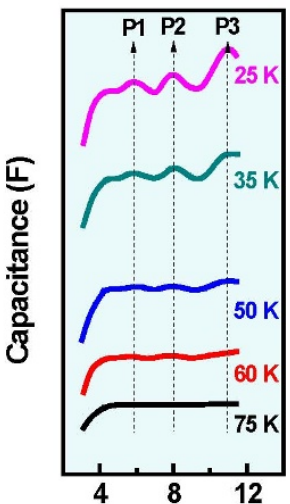

Gate Voltage (V)

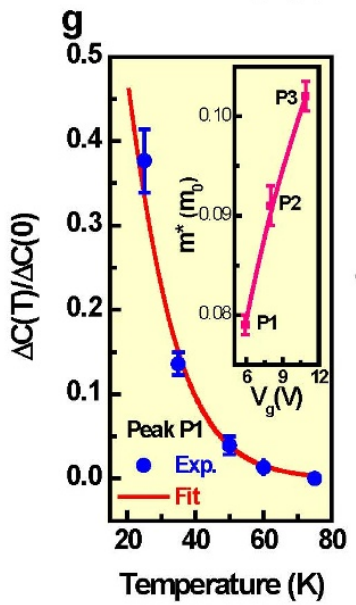

k

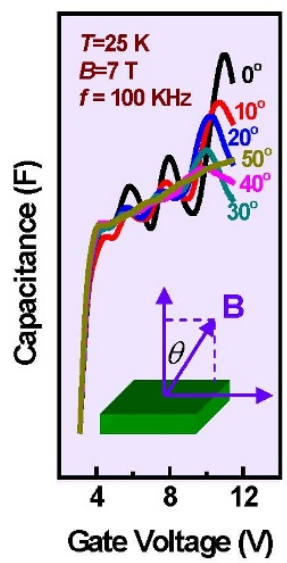

d

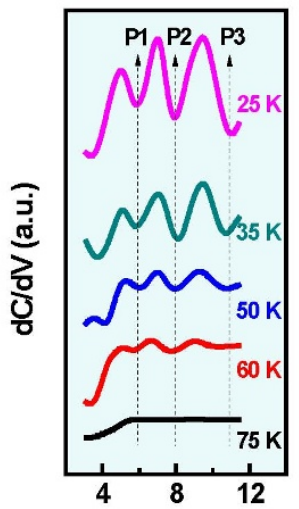

h

Gate Voltage (M)

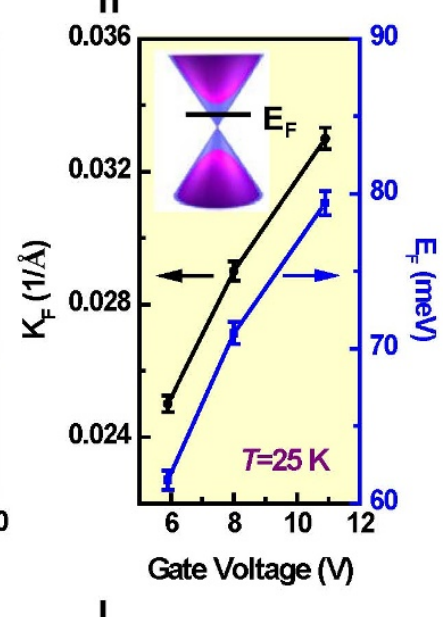

I

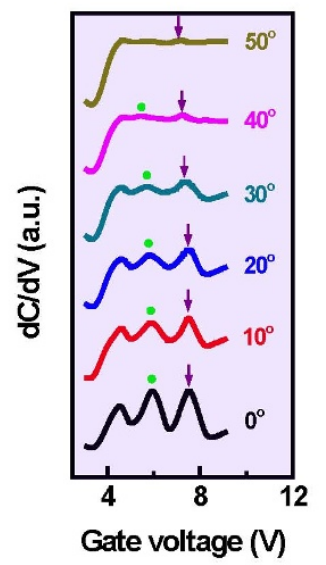

Figure $4 \mid$ Quantitative analysis of quantum capacitance in $\mathrm{Bi}_{2} \mathrm{Se}_{3}$. (a, b) Magnetic-field- and frequency-dependent capacitance at $T=25 \mathrm{~K}$ and $f=100 \mathrm{KHz}$. (e, f) and (i,j) The same measurements when the temperature is increased to 35 and $50 \mathrm{~K}$, respectively. $C_{Q}$ demonstrates a strong frequency dependence at all three temperatures. (c) Temperature-dependent capacitance at $B=7 \mathrm{~T}$ and $f=100 \mathrm{KHz}$. Three oscillation peaks are clearly identified, denoted as P1, P2 and P3. (d) The first derivative of the total capacitance as a function of temperature. P1, P2 and P3 correspond to the three valleys of the peaks because of a $90^{\circ}$ phase shift compared with those in (c). (g) Extraction of the cyclotron effective mass by taking the temperature-dependent oscillation amplitude in (d). Three effective masses for peaks P1-P3 were obtained in the inset. (h) Fermi vector $k_{F}$ and Fermi energy $E_{F}$ as a function of gate voltage. The inset describes the calculated positions of the Fermi level relative to the Dirac point. (k) The total capacitance as a function of the measurement angles. The inset depicts the measurement setup, in which the device was rotated from 0 to $50^{\circ}$. (i) The first derivative of the total capacitance as a function of the rotation angle. The traces of arrows (in purple) and dots (in green) suggest the change of the oscillation frequencies. The curves in all the figures except for $(\mathrm{g}),(\mathrm{h})$ and $(\mathrm{k})$ are vertically shifted for clarity. 


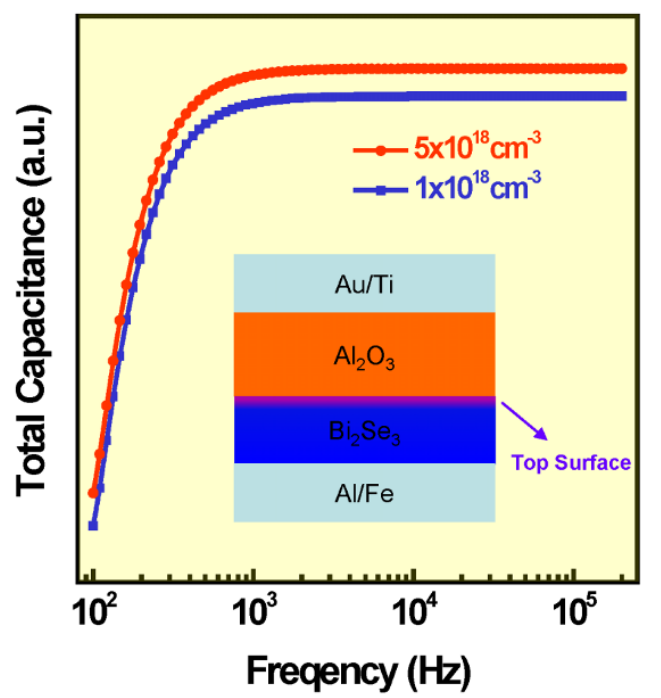

Figure 5 Simulated frequency-dependent total capacitance. A device model from the inset is constructed in accordance with the experimental setup (Figs. 1d and 1e). During simulations, only the top surface of $\mathrm{Bi}_{2} \mathrm{Se}_{3}$ is considered. At low frequencies, the total capacitance exhibits a strong dependence on frequency, primarily attributed to the sufficient responses for the bulk and the surface carriers. When the frequency is set beyond $1 \mathrm{KHz}$, however, a desirable surface-dominant regime is attained, in which the bulk interference is reduced at elevated frequencies. The inset depicts the device structure used for the simulations.

verified by an ambipolar field effect in quantum capacitance (Supplementary Fig. S11).

\section{Discussion}

To understand the frequency dependence of quantum oscillations, we carried out qualitative simulations using Technology Computer Aided Design (TCAD). A capacitor device was constructed by considering the experimental conditions. Figure 5 shows that the total capacitance changes with the frequency of the ac signals. In essence, the capacitance for the system depends on the total available charges under specific measurement frequencies ${ }^{49,51}$. In the low-frequency regime $(f<1 \mathrm{KHz}$, Fig. 5), both the bulk and surface carriers can respond to the external $a c$ signals, and the system can be represented by a series capacitor model $\left(1 / C_{T}=1 / C_{O X}+1 / C_{Q}+1 / C_{B}\right)$. The bulk interference exists in the system, and the quantum capacitance can hardly make itself distinguishable. In the high-frequency regime $(f>$ $1 \mathrm{KHz}$, Fig. 5), however, the available charges accounting for the total capacitance primarily originate from the surface states because the bulk carriers do not have adequate time to respond to the high ac frequencies $^{51-54}$. Thus, the elimination of the bulk term leads to a significant manifestation of the surface quantum capacitance (1/ $\left.C_{T}=1 / C_{O X}+1 / C_{Q}\right)$. By taking two different surface carrier densities (Fig. 5, red and blue curves), we can verify the effect of the available surface charges on the total capacitance. In short, our simulations provide sensible explanations for the observed frequency dispersion of the total capacitance, although a detailed theoretical treatise is still needed. The discovery of the quantum capacitance in topological insulators may facilitate future progress toward the creation of topological devices by probing high-temperature surface states and incorporating gated structures.

\section{Methods}

MBE growth. Thin film growth was carried out with an ultra-high vacuum Perkin Elmer MBE system. Si (111) substrates were cleaned using the standard Radio Corporation of America (RCA) procedure before being transferred into the growth chamber. High-purity Bi (99.9999\%) and Se (99.99\%) elementary sources were evaporated by conventional effusion cells. During growth, the Bi and Se cells were kept at 490 and $200^{\circ} \mathrm{C}$, respectively, while the Si (111) substrate was kept at $150^{\circ} \mathrm{C}$ (growth temperature). The epitaxial growth was monitored using the in-situ RHEED technique, and the surface was observed to be atomically flat, as evidenced by the streaky RHEED patterns (Fig. 1a). Digital images of the RHEED were captured using a KSA400 system made by K-space Associate, Inc.

Characterizations. (1) ARPES. High-resolution ARPES experiments were performed at beam line 12.0.1 of the Advanced Light Source at Lawrence Berkeley National Laboratory, using $100 \mathrm{eV}$ and $52 \mathrm{eV}$ photons to measure the core levels and surface states, respectively. (2) TEM. High-resolution TEM experiments were performed on a Philips Tecnai F20 (S)TEM operating at $200 \mathrm{KV}$. The digital images were recorded by a Gatan ${ }^{\circledR} 2 \mathrm{k} \times 2 \mathrm{k}$ CCD camera. (3) Capacitance measurements. Frequency-dependent capacitance measurements were conducted with an HP/ Agilent 4274A multi-frequency LCR meter. The ac frequency could be tuned from $100 \mathrm{~Hz}-100 \mathrm{KHz}$, while a $d c$ bias can be applied from 0 to $\pm 35 \mathrm{~V}$. The LCR meter was connected to a physical property measurement system (PPMS), where the devices were located. We were also able to systematically vary several experimental variables such as the temperature, magnetic field, measurement frequency, and external gate bias. Multiple lock-in-amplifiers and Keithley source meters were also connected to the PPMS system, enabling comprehensive and high-sensitivity transport measurements for the top-gated Hall bar and capacitor devices.

Device fabrication. The $\mathrm{MBE}$-grown $\mathrm{Bi}_{2} \mathrm{Se}_{3}$ thin film $(\sim 10 \mathrm{~nm})$ was patterned into a micron-scale Hall bar geometry using conventional optical photolithography and a subsequent $\mathrm{CHF}_{3}$ dry-etching of $18 \mathrm{~s}$. A 15-nm-thick, high- $\kappa \mathrm{Al}_{2} \mathrm{O}_{3}$ dielectric layer was deposited using atomic layer deposition (ALD). Hall channel contacts were defined by $e$-beam evaporation after etching away $\mathrm{Al}_{2} \mathrm{O}_{3}$ in the contact areas. A metal stack of Fe/Al $(20 \mathrm{~nm} / 100 \mathrm{~nm})$ was directly deposited onto the exposed $\mathrm{Bi}_{2} \mathrm{Se}_{3}$ surface. A top-gate metal scheme of Ti/Au $(10 \mathrm{~nm} / 90 \mathrm{~nm})$ was achieved using a second step of photolithography and $e$-beam evaporation.

Device simulation. We used a visual TCAD software package (version 1.7.3) from Cogenda.com. The simulated device structure is depicted in Fig. 5 inset. The topological surface is assumed to have a depth of $1 \mathrm{~nm}$ and carrier densities of $1 \times 10^{18} \mathrm{~cm}^{-3}$ and $5 \times 10^{18} \mathrm{~cm}^{-3}$ (two scenarios). Bulk $\mathrm{Bi}_{2} \mathrm{Se}_{3}$ has a carrier density of $6.5 \times 10^{19}-1.3 \times 10^{20} \mathrm{~cm}^{-3}$, attributed to the formation of an impurity band as elaborated in Supplementary Fig. S6. Our simplified model describes the physics of frequency-dependent capacitance in a qualitative manner. The absolute values of the total capacitance can be varied by altering the above parameters; however, the underlying physics remains the same, which forms the basis of our simulations.

1. Kane, C. L. \& Mele, E. J. Topological order and the quantum spin Hall effect. Phys. Rev. Lett. 95, 146802 (2005).

2. Zhang, H. et al. Topological insulators in $\mathrm{Bi}_{2} \mathrm{Se}_{3}, \mathrm{Bi}_{2} \mathrm{Te}_{3}$ and $\mathrm{Sb}_{2} \mathrm{Te}_{3}$ with a single Dirac cone on the surface. Nat. Phys. 5, 438-442 (2009).

3. Moore, J. Topological insulators: The next generation. Nat. Phys. 5, 378-380 (2009).

4. Fu, L. \& Kane, C. L. Topological insulators with inversion symmetry. Phys. Rev. B 76, 045302 (2007).

5. Qu, D.-X., Hor, Y. S., Xiong, J., Cava, R. J. \& Ong, N. P. Quantum oscillations and Hall anomaly of surface states in the topological insulator $\mathrm{Bi}_{2} \mathrm{Te}_{3}$. Science $\mathbf{3 2 9}$, 821-824 (2010).

6. Xia, Y. et al. Observation of a large-gap topological-insulator class with a single Dirac cone on the surface. Nat. Phys. 5, 398-402 (2009).

7. Zhang, T. et al. Experimental demonstration of topological surface states protected by time-reversal symmetry. Phys. Rev. Lett. 103, 266803 (2009).

8. Alpichshev, Z. et al. STM imaging of electronic waves on the surface of $\mathrm{Bi}_{2} \mathrm{Te}_{3}$ :topologically protected surface states and hexagonal warping effects. Phys. Rev. Lett. 104, 016401 (2010).

9. Veldhorst, M. et al. Josephson supercurrent through a topological insulator surface state. Nat Mater advance online publication (2012).

10. McIver, J. W., Hsieh, D., Steinberg, H., Jarillo Herrero, P. \& Gedik, N. Control over topological insulator photocurrents with light polarization. Nat Nano 7, 96-100 (2012).

11. Cho, S. et al. Topological insulator quantum dot with tunable barriers. Nano Letters 12, 469-472 (2012).

12. Bahramy, M. S., Yang, B. J., Arita, R. \& Nagaosa, N. Emergence of noncentrosymmetric topological insulating phase in BiTeI under pressure. Nat Commun 3, 679 (2012).

13. Arakane, T. et al. Tunable Dirac cone in the topological insulator Bi2-xSbxTe3ySey. Nat Commun 3, 636 (2012).

14. Qi, X. L., Hughes, T. L. \& Zhang, S. C. Topological field theory of time-reversal invariant insulators. Phys. Rev. B 78, 195424 (2008).

15. Bernevig, B. A., Hughes, T. L. \& Zhang, S.-C. Quantum spin Hall effect and topological phase transition in HgTe quantum wells. Science 314, 1757-1761 (2006).

16. Konig, M. et al. Quantum spin hall insulator state in HgTe quantum wells. Science 318, 766-770 (2007).

17. Zhang, Y. et al. Crossover of the three-dimensional topological insulator $\mathrm{Bi}_{2} \mathrm{Se}_{3}$ to the two-dimensional limit. Nat. Phys. 6, 584-588 (2010). 
18. Kou, X. F. et al. Epitaxial growth of high mobility $\mathrm{Bi}_{2} \mathrm{Se}_{3}$ thin films on CdS. Appl. Phys. Lett. 98, 242102 (2011).

19. Bansal, N. et al. Epitaxial growth of topological insulator Bi2Se3 film on $\mathrm{Si}(111)$ with atomically sharp interface. Thin Solid Films 520, 224-229 (2011).

20. Li, H. D. et al. The van der Waals epitaxy of $\mathrm{Bi}_{2} \mathrm{Se}_{3}$ on the vicinal $\mathrm{Si}(111)$ surface: an approach for preparing high-quality thin films of a topological insulator. New Journal of Physics 12, 103038 (2010).

21. Zhang, J. et al. Band structure engineering in $\left(\mathrm{Bi}_{1-\mathrm{x}} \mathrm{Sb}_{\mathrm{x}}\right)_{2} \mathrm{Te}_{3}$ ternary topological insulators. Nat Commun 2, 574 (2011).

22. Brahlek, M., Kim, Y. S., Bansal, N., Edrey, E. \& Oh, S. Surface versus bulk state in topological insulator $\mathrm{Bi}_{2} \mathrm{Se}_{3}$ under environmental disorder. Appl. Phys. Lett. 99, 012109 (2011)

23. Taskin, A. A., Ren, Z., Sasaki, S., Segawa, K. \& Ando, Y. Observation of Dirac Holes and Electrons in a Topological Insulator. Phys. Rev. Lett. 107 (2011).

24. Kong, D. \& Cui, Y. Opportunities in chemistry and materials science for topological insulators and their nanostructures. Nat Chem 3, 845-849 (2011).

25. Xiu, F. Manipulating surface states in topological insulator nanoribbons. Nature Nanotech. 6, 216-221 (2011)

26. Peng, H. et al. Topological insulator nanostructures for near-infrared transparent flexible electrodes. Nat Chem advance online publication (2012).

27. Chen, Y. L. et al. Massive Dirac Fermion on the Surface of a Magnetically Doped Topological Insulator. Science 329, 659-662 (2010).

28. Bianchi, M. et al. Coexistence of the topological state and a two-dimensional electron gas on the surface of Bi2Se3. Nat Commun 1, 128 (2010).

29. Cheng, P. et al. Landau quantization of topological surface states in $\mathrm{Bi}_{2} \mathrm{Se}_{3}$. Phys. Rev. Lett. 105, 076801 (2010).

30. Steinberg, H., Gardner, D. R., Lee, Y. S. \& Jarillo-Herrero, P. Surface State Transport and Ambipolar Electric Field Effect in $\mathrm{Bi}_{2} \mathrm{Se}_{3}$ Nanodevices. Nano Letters 10, 5032-5036 (2010).

31. Analytis, J. G. et al. Two-dimensional surface state in the quantum limit of a topological insulator. Nat. Phys. 6, 960-964 (2010).

32. Sacépé, B. et al. Gate-tuned normal and superconducting transport at the surface of a topological insulator. Nat Commun 2, 575 (2011)

33. Chen, J. et al. Gate-Voltage Control of Chemical Potential and Weak Antilocalization in $\mathrm{Bi}_{2} \mathrm{Se}_{3}$. Phys. Rev. Lett. 105, 176602 (2010).

34. Ponomarenko, L. A. et al. Density of States and Zero Landau Level Probed through Capacitance of Graphene. Phys. Rev. Lett. 105, 136801 (2010)

35. Luryi, S. Quantum capacitance devices. Appl. Phys. Lett. 52, 501-503 (1988)

36. Xu, H. et al. Quantum Capacitance Limited Vertical Scaling of Graphene FieldEffect Transistor. ACS Nano 5, 2340-2347 (2011).

37. Xia, J., Chen, F., Li, J. \& Tao, N. Measurement of the quantum capacitance of graphene. Nat Nano 4, 505-509 (2009).

38. Ilani, S., Donev, L. A. K., Kindermann, M. \& McEuen, P. L. Measurement of the quantum capacitance of interacting electrons in carbon nanotubes. Nat Phys $\mathbf{2}$, 687-691 (2006).

39. John, D. L., Castro, L. C. \& Pulfrey, D. L. Quantum capacitance in nanoscale device modeling. J. Appl. Phys. 96, 5180-5184 (2004).

40. Giannazzo, F., Sonde, S., Raineri, V. \& Rimini, E. Screening Length and Quantum Capacitance in Graphene by Scanning Probe Microscopy. Nano Letters 9, 23-29 (2008).

41. Guo, J., Yoon, Y. \& Ouyang, Y. Gate Electrostatics and Quantum Capacitance of Graphene Nanoribbons. Nano Letters 7, 1935-1940 (2007).

42. Henriksen, E. A. \& Eisenstein, J. P. Measurement of the electronic compressibility of bilayer graphene. Phys. Rev. B 82, 041412 (2010).

43. Droscher, S. et al. Quantum capacitance and density of states of graphene. Appl. Phys. Lett. 96, 152104 (2010).

44. Cho, S., Butch, N. P., Paglione, J. \& Fuhrer, M. S. Insulating Behavior in Ultrathin Bismuth Selenide Field Effect Transistors. Nano Letters 11, 1925-1927 (2011).

45. Yu, R. et al. Quantized Anomalous Hall Effect in Magnetic Topological Insulators. Science 329, 61-64 (2010).

46. Kim, Y. S. et al. Thickness-dependent bulk properties and weak antilocalization effect in topological insulator Bi_\{2\}Se_\{3\}. Phys. Rev. B 84, 073109 (2011).
47. Hsieh, D. et al. A tunable topological insulator in the spin helical Dirac transport regime. Nature 460, 1101-1105 (2009).

48. Kordyuk, A. A. et al. Photoemission-induced gating of topological insulators. Phys. Rev. B 83, 081303 (2011).

49. Sze, S. Physics of Semiconductor Devices, 3rd ed. (Wiley, New York, 2007).

50. Lind, E., Niquet, Y.-M., Mera, H. \& Wernersson, L.-E. Accumulation capacitance of narrow band gap metal-oxide-semiconductor capacitors. Appl. Phys. Lett. 96, 233507 (2010).

51. Bülbül, M. M. \& Zeyrek, S. Frequency dependent capacitance and conductancevoltage characteristics of $\mathrm{Al} / \mathrm{Si}_{3} \mathrm{~N}_{4} / \mathrm{p}$-Si(100) MIS diodes. Microelectronic Engineering 83, 2522-2526 (2006).

52. Lieneweg, U. Frequency response of charge transfer in MOS inversion layers. Solid-State Electronics 23, 577-583 (1980).

53. Xuan, Y., Lin, H. C., Ye, P. D. \& Wilk, G. D. Capacitance-voltage studies on enhancement-mode InGaAs metal-oxide-semiconductor field-effect transistor using atomic-layer-deposited $\mathrm{Al}[\mathrm{sub} 2] \mathrm{O}$ [sub 3] gate dielectric. Appl. Phys. Lett. 88, 263518 (2006).

54. Jiang, J., Awadelkarim, O. O., Lee, D. O., Roman, P. \& Ruzyllo, J. On the capacitance of metal/high-k dielectric material stack/silicon structures. SolidState Electronics 46, 1991-1995 (2002).

55. Zhang, Y., Tan, Y.-W., Stormer, H. L. \& Kim, P. Experimental observation of the quantum Hall effect and Berry's phase in graphene. Nature 438, 201-204 (2005).

56. Liao, L. et al. High-speed graphene transistors with a self-aligned nanowire gate. Nature 467, 305-308 (2010).

57. Fang, T., Konar, A., Xing, H. \& Jena, D. Carrier statistics and quantum capacitance of graphene sheets and ribbons. Appl. Phys. Lett. 91, 092109 (2007).

\section{Acknowledgements}

F.X. would like to acknowledge the financial support received from the National Science Foundation under the Award No. 1201883, and the College of Engineering at Iowa State University. The Microelectronics Research Center at Iowa State provided substantial equipment supports during the project. F.X., K.W. Y.W. and J.Z. would like to thank the Focus Center Research Program-Center on Functional Engineered Nano Architectonics (FENA), the Australia Research Council. Y.W. would like to thank National Science Foundation of China (No. 11174244) for the financial support received for this project. F.X. would like to acknowledge Mingqiang Bao for setting up the CV measurement system, Peng Zhang for helpful discussions on ARPES, and Shen Chen for extensive guidance on the usage of the Visual TCAD software.

\section{Author contributions}

F.X. conceived and designed the research. F.X., X.K. and M.L. fabricated the capacitor devices and performed the measurements. L.H., M.L. and X.Y. contributed to the measurements and analysis. Y.W. and J.Z. performed structural analysis. A.V. provided the ARPES measurements. X.K. and F.X. performed numerical simulations on capacitances. F.X., N.M. and X.K. wrote the paper, with help from all the other co-authors.

\section{Additional information}

Reprints and permission information is available online at http://npg.nature.com/ reprintsandpermissions.

Supplementary information accompanies this paper at http://www.nature.com/ scientificreports

Competing financial interests: The authors declare no competing financial interests.

License: This work is licensed under a Creative Commons

Attribution-NonCommercial-ShareAlike 3.0 Unported License. To view a copy of this license, visit http://creativecommons.org/licenses/by-nc-sa/3.0/

How to cite this article: Xiu, F. et al. Quantum Capacitance in Topological Insulators. Sci. Rep. 2, 669; DOI:10.1038/srep00669 (2012). 